PROCEEDINGS OF THE

AMERICAN MATHEMATICAL SOCIETY

Volume 127, Number 9, Pages 2535-2546

S 0002-9939(99)05429-5

Article electronically published on May 19, 1999

\title{
UNIFORM BOUNDS FOR STABLY INTEGRAL POINTS ON ELLIPTIC CURVES
}

\author{
PATRICIA L. PACELLI
}

(Communicated by Ron Donagi)

\begin{abstract}
We show that a conjecture by Lang and Vojta regarding integral points on varieties of logarithmic general type implies the existence of a uniform bound on the number of stably $S$-integral points on an elliptic curve over a degree- $d$ number field.
\end{abstract}

\section{INTRODUCTION}

Many recent results have shown how various conjectures in arithmetic geometry imply uniform boundedness results for points on curves. S. Lang [L] has conjectured that the rational points on a variety of general type are not Zariski dense, a conjecture which can be viewed as a generalization to higher dimensions of the Mordell Conjecture. L. Caporaso, J. Harris, and B. Mazur [CHM] use this conjecture to prove a fascinating result; they show that Lang's conjecture implies a uniform bound on the number of rational points on a curve of genus bigger than 1 over a fixed number field. In $[\mathrm{P}]$, the author shows that this bound depends solely on the genus and the degree of the number field, extending a result by D. Abramovich ([A1]).

Abramovich has also shown that a conjecture made by Lang and P. Vojta [V] regarding the non-denseness of integral points on varieties of logarithmic general type implies a result similiar to the $[\mathrm{CHM}]$ result, but for stably integral points on elliptic curves. More precisely, let $X$ be a variety of logarithmic general type (see Definition 1.5) defined over a number field $K$. Let $S$ be a finite set of places of $K$ and let $\mathcal{O}_{K, S}$ be the ring of $S$-integers. If $\mathcal{X}$ is a model of $X$ over $\operatorname{Spec}\left(\mathcal{O}_{K, S}\right)$, Lang and Vojta conjecture that the set of $S$-integral points of $\mathcal{X}$ is not Zariski dense, a generalization of both Mordell's conjecture and Siegel's theorem. Now, let $E$ be an elliptic curve defined over a number field $K$, with $S$ as above. A point $P \in E(K)$ is said to be stably $S$-integral, i.e. $P \in E(K, S)$, if $P$ is $S$-integral on the complement of the origin after semistable reduction (see Definition 1.7 for a more precise statement). In [A2], Abramovich shows that a consequence of the Lang-Vojta conjecture is the existence of a uniform bound, depending on $K$ and $S$, on the number of stably $S$-integral points of $E$. In this paper we show that,

Received by the editors September 2, 1997.

1991 Mathematics Subject Classification. Primary 11Gxx, 14Gxx.

It is a pleasure to thank Dan Abramovich and Glenn Stevens for encouraging me to pursue this work. Dan Abramovich was also kind enough to read earlier versions of this paper and provide extremely helpful comments.

(C)1999 American Mathematical Society 
once again, this bound is independent of the actual number field and, in fact, only depends on $S$ and the degree of $K$. Our main theorem is the following:

Theorem 1.1. Assume the Lang-Vojta conjecture. Fix an integer $d>0$ and fix a finite set of places $S_{0}$ in $\mathbf{Q}$ containing 3. Then there exists a bound $N\left(d, S_{0}\right)$ depending on $d$ and $S_{0}$, such that for any number field $K$ of degree $d$, for any finite set $S$ of places of $K$ lying over $S_{0}$, and for any elliptic curve $E$ defined over $K$, it follows that $|E(K, S)| \leq N\left(d, S_{0}\right)$, where $E(K, S)$ denotes the set of stably $S$-integral points of $E$.

If the elliptic curve $E$ with which we start is actually semistable, then stably $S$ integral points are simply $S$-integral points. This leads immediately to the following corollary, just as in [A2]:

Corollary 1.2. Assume the Lang-Vojta conjecture. Fix an integer $d>0$ and fix a finite set of places $S_{0}$ in $\mathbf{Q}$ containing 2 and 3. Then there exists a bound $N\left(d, S_{0}\right)$, depending on $d$ and $S_{0}$, such that for any number field $K$ of degree $d$, for any finite set $S$ of places of $K$ lying over $S_{0}$, and for any semistable elliptic curve $E$ defined over $K$, it follows that $\left|E\left(\mathcal{O}_{K, S}\right)\right| \leq N\left(d, S_{0}\right)$.

We have another corollary, which is that the Lang-Vojta conjecture combined with Theorem 1.1 implies L. Merel's theorem on uniform boundedness for torsion points on elliptic curves. Of course, Merel's theorem is unconditional, but it is perhaps interesting to see here the connections with Lang-Vojta and stably integral points. More specifically, we have:

Corollary 1.3. Assume the Lang-Vojta conjecture. Fix a positive integer d, and let $E / K$ be an elliptic curve over any number field $K$ of degree $d$. Then there exists a bound $N(d)$ on the order of the group of torsion points of $E(K)$.

Proof. By $[\mathrm{KM}]$, it is enough to bound the number of torsion points of prime order on any elliptic curve $E$ over any number field $K$ of degree $d$. We can add level 3 structure to $E$, producing a field extension $L$ of $K$ of bounded degree; say $[L: K] \leq B$, where $E / L$ has semistable reduction over all primes $p>3$. Define a set $S$ as follows:

$$
S=\left\{v \in M_{K}^{0} \mid \operatorname{ord}_{v}(p) \geq p-1 \text { for some primes } p \text { such that } p-1 \leq B \cdot d\right\},
$$

where $T$ is the set of places of $L$ lying over $S$ and over the primes 2 and 3 . To see this, first, suppose that $p-1>B \cdot d$. Then if $P$ is not integral in $E(L)$ for some place $v$, it follows from Theorem VIII.7.1 in [S] that $\operatorname{ord}_{v}(p) \geq p-1>B \cdot d$. This is

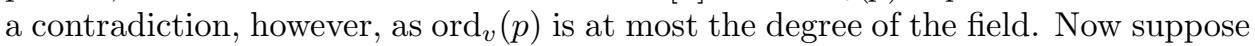
that $p-1 \leq B \cdot d$. If $P$ is not integral for some place $v$, it follows from above that $v \in T$.

The above claim says that torsion points of prime order in $E(K)$ are stably $S$-integral, where the set $S$ depends on the degree $d$. But Theorem 1.1 implies that there is a bound, $N(d)$, on the number of such points, and so the corollary is proven.

The methods used in this paper to prove Theorem 1.1 are very close to those of $[\mathrm{P}]$, and much of the definitions and notation will be similar, as we show that the ideas of that paper apply in this new setting. 
Recall the following definitions:

Definition 1.4. A line bundle $L$ on a variety $X$ is big if for high values of $k$, global sections of $H^{0}\left(X, L^{\otimes k}\right)$ determine a birational map of $X$ into projective space.

Definition 1.5. Let $X$ be a quasi-projective variety over C. Let $f: Y \rightarrow X$ be a resolution of singularities, so that $f$ is a proper, birational morphism, and $Y$ is a smooth variety. Let $Y \subset \bar{Y}$ be a projective compactification such that $\bar{Y}$ is smooth, and the reduced variety $D=\bar{Y} \backslash Y$ is a divisor of normal crossings. Let $\omega_{\bar{Y}}$ be the dualizing sheaf of $\bar{Y}$. Then $X$ is said to be of logarithmic general type if $\omega_{\bar{Y}}(D)$ is big.

Let $X$ be a quasi-projective variety of logarithmic general type defined over a number field $K$. Let $S$ be a finite set of places of $K$, and let $R$ be the ring of $S$ integers. Choose a model $\mathcal{X}$ of $X$ over $R$. The following is a conjecture made by Lang and Vojta.

Conjecture 1.6 (Lang-Vojta). The set of integral points $\mathcal{X}(R)$ is not Zariski dense in $\mathcal{X}$.

We now give a precise definition of stably $S$-integral points:

Definition 1.7. Let $E / K$ be an elliptic curve, $S$ a finite set of places of $K$, and $P \in E(K)$. Assume $E$ admits a semistable model $\mathcal{E}$ over $\mathcal{O}_{L, T}$, where $L$ is a finite extension of $K$ and $T$ is the set of places of $L$ above $S$. Let $\mathcal{E}_{0}=\mathcal{E} \backslash 0$. We say $P$ is stably $S$-integral, denoted $P \in E(K, S)$, if $P \in \mathcal{E}_{0}\left(\mathcal{O}_{L, T}\right)$, i.e., if $P$ is $T$-integral on the complement of the origin of the semistable model $\mathcal{E}$.

Remark 1.8. Suppose that $S$ contains all places of $K$ dividing 2 and 3 , and that $v \notin S$ is a place at which $E$ has additive reduction. Then $P \in E(K)$ is stably $S$-integral if $P$ does not go through the zero component of the Néron model of $E$ at $v$. (See [A2], section 4, for a discussion of stably $S$-integral points in terms of stably minimal models of elliptic curves.)

Remark 1.9. After adding level 3 structure, an elliptic curve admits semistable reduction away from the prime 3 . Thus, a point $P \in E(K)$ is stably $S$-integral if it is $S$-integral on the Néron model after adding level 3 structure to $E$. Note also that stably $S$-integral points remain stably $S$-integral after field extensions.

Example 1.10. Let $E / \mathbf{Q}$ be the curve given by

$$
y^{2}=x^{3}+5 \text {. }
$$

Let $S=\{2,3\}$, and let $P=(-1,2)$. The curve $E$ has additive reduction at the prime 5 , but making the substitution

$$
x=\sqrt[3]{5} x^{\prime}, \quad y=\sqrt{5} y^{\prime},
$$

we obtain a semistable model of $E$ over $L=\mathbf{Q}(\sqrt[6]{5})$ given by

$$
\mathcal{E}: y^{\prime 2}=x^{\prime 3}+1
$$

Under this change of variables, the point $P$ is sent to the point $\left(\frac{-1}{\sqrt[3]{5}}, \frac{2}{\sqrt{5}}\right)$. Therefore, if $T$ is the set of places of $L$ lying over $S$, we see that $P$ is not $T$-integral, and so $P$ is not a stably $S$-integral point of $E$. 
Example 1.11. Let $E / \mathbf{Q}$ be the curve given by

$$
E: y^{2}=x^{3}+5 x,
$$

and let $S=\{2,3\}$ and $P=(20,90)$. Once again, $E$ has additive reduction at 5 , but if we take $a=\sqrt{-5}$ and apply the change of coordinates:

$$
x=a x^{\prime}, \quad y=a^{\frac{3}{2}} y^{\prime},
$$

we obtain a semistable model for $E$ over the field $L=\mathbf{Q}(\sqrt{a})$ :

$$
\mathcal{E}: y^{\prime 2}=x^{\prime 3}-x^{\prime}
$$

Under the above change of variables, the point $P$ is sent to the point $(-4 a, 18 \sqrt{a})$, which is integral. Thus, $P$ is stably $S$-integral on $E$.

In [RS], K. Rubin and A. Silverberg study the universal family $E_{1} \rightarrow \mathbf{P}^{1}$ of semistable elliptic curves over $\mathbf{C}$ with full level 3 structure. The surface $E_{1}$ can be identified with the total space of the elliptic pencil:

$$
\lambda\left(X^{3}+Y^{3}+Z^{3}\right)-3 \mu X Y Z=0,
$$

which maps to $\mathbf{P}^{1}$ via $[\lambda: \mu]$. We choose the section of the pencil over the point $[X: Y: Z]=[1:-1: 0]$ as the origin of the elliptic surface, and denote this origin by $\Theta$.

Remark 1.12. Given any elliptic curve $E / K$ as in Theorem 1.1, it is possible to add level 3 structure by replacing $K$ with an extension of bounded degree. This ensures that $E$ now appears as a fiber of the family $E_{1}$, and, moreover, does not affect our result, since the bound in Theorem 1.1 depends on the degree of the number field. (A more detailed argument of a similar situation in the case of $d$-gonal curves is given in section 5 of $[\mathrm{P}]$.)

Fix a positive integer $d$ and a finite set of places $S_{0}$ of $\mathbf{Q}$ as in the statement of Theorem 1.1.

We fix an integer $n$, and let $E_{n} \rightarrow \mathbf{P}^{1}$ be the $n$-th fibered product of $E_{1}$ over $\mathbf{P}^{1}: E_{n}=\left(E_{1}\right)_{\mathbf{P}^{1}}^{n}$. We will be working with subvarieties of $E_{n}^{d}$, where $E_{n}^{d} \rightarrow\left(\mathbf{P}^{1}\right)^{d}$. Denote by $Y_{n}$ the symmetric product $\operatorname{Sym}^{d}\left(E_{n}\right)$.

For $i=1, \ldots, d$ and for $j=1, \ldots, n$, we have projections

$$
\begin{aligned}
f_{i}: E_{n}^{d} & \rightarrow E_{n} \\
h_{j}: E_{n} & \rightarrow E_{1} .
\end{aligned}
$$

Let $\Theta_{n}$ be the divisor on $E_{n}^{d}$ which comes from the pullbacks of the origin $\Theta$ :

$$
\Theta_{n}=\sum_{i=1}^{d} \sum_{j=1}^{n} f_{i}^{*} h_{j}^{*}(\Theta) .
$$

Similarly, for any positive integer $k$ we will denote by $\Theta_{n+k}$ the corresponding divisor on $E_{n+k}^{d}$. We will let $\Theta_{Y_{n}}$ denote the image of $\Theta_{n}$ in the quotient $Y_{n}$.

Suppose $K$ is any number field of degree $d, S$ a finite set of places of $K$ lying over $S_{0}$, and $E$ an elliptic curve defined over $K$. Let $\sigma_{1}, \ldots, \sigma_{d}$ denote the $d$ embeddings of $K$ into $\bar{K}$. Suppose that $P_{1}, \ldots, P_{n}$ are in $E(K, S)$. Recall that this implies the existence of a field extension $L$ of $K$, and a set of places $T$ lying over $S$, along with a semistable model $\mathcal{E}$ of $E$ over $\mathcal{O}_{L, T}$, such that $P_{i} \in(\mathcal{E} \backslash 0)\left(\mathcal{O}_{L, T}\right)$ for each $i$. By Remark 1.12 we may assume that $E / L$ has level 3 structure and appears as a fiber of the surface $E_{1}$. 
It follows that the $n$-tuple $\left(P_{1}, \ldots, P_{n}\right)$ of points of $E(K, S)$ gives rise to a $T$-integral point on $E_{n} \backslash\left(\sum_{j=1}^{n} h_{j}^{*} \Theta\right)$, and hence also yields a $T$-integral point $y_{P_{1}, \ldots, P_{n}} \in Y_{n} \backslash \Theta_{Y_{n}}:$

$$
y_{P_{1}, \ldots, P_{n}}=\left\{\left(P_{1}, \ldots P_{n}\right)^{\sigma_{1}}, \ldots,\left(P_{1}, \ldots P_{n}\right)^{\sigma_{d}}\right\} \text {. }
$$

Definition 1.13. A point $y_{P_{1}, \ldots, P_{n}} \in Y_{n} \backslash \Theta_{Y_{n}}$ is said to be off-diagonal if the points $P_{i} \in E(K, S)$ are pairwise distinct.

Definition 1.14. Choose $m \geq n$. An off-diagonal integral point $y_{\left(P_{1}, \ldots, P_{n}\right)} \in Y_{n}$ is $m$-prolongable if :

1. There exists an off-diagonal point $y_{\left(P_{1}, \ldots, P_{m}\right)} \in Y_{m} \backslash \Theta_{Y_{m}}$ such that the restriction of $y_{\left(P_{1}, \ldots, P_{m}\right)}$ to the first $n$ coordinates yields $y_{\left(P_{1}, \ldots, P_{n}\right)}$.

2. Each $P_{i}, i=1, \ldots, m$, is defined over the same number field $K$ of degree $d$, but is not defined over any smaller field.

We will call $E_{n}^{(m)}$ the set of $m$-prolongable points in $Y_{n} \backslash \Theta_{Y_{n}}$, and will denote by $F_{n}^{(m)}$ the Zariski closure $\overline{E_{n}^{(m)}}$. Let $F_{n}=\bigcap_{m>n} F_{n}^{(m)}$, and let $\Theta_{F_{n}}$ be the restriction of $\Theta_{Y_{n}}$ to $F_{n}$. We will later denote by $\Theta_{F_{n+k}}$ the restriction of $\Theta_{Y_{n+k}}$ to $F_{n+k}$. Note that by the Noetherian property of the Zariski topology, we know that $F_{n}=F_{n}^{\left(m_{0}\right)}$ for some $m_{0}$.

I claim that our goal is to show that, for some $n, F_{n}$ is empty. A short argument shows that this suffices to prove Theorem 1.1: whenever a fiber of $E_{1}$ contains a large number of integral points, say $m$, then for any $n<m$, it follows that $E_{n}^{(m)}$ is non-empty. If Theorem 1.1 is not true, then $m$ grows without bound as one varies the fibers of $E_{1}$, implying that for arbitrarily large $n$, the $F_{n}$ 's will be non-empty. Moreover, this implies that for all smaller $n$ 's the $F_{n}$ 's will also be non-empty, due to the surjectivity of the natural projection maps $F_{n+1} \rightarrow F_{n}$.

Just as in $[\mathrm{P}]$, Lemmas 2.6 and 2.7, the projection $F_{n+1} \rightarrow F_{n}$ is surjective, and each component of $F_{n}$ has a component of $F_{n+1}$ above it of positive relative dimension. We will perform an induction argument on the relative dimension of $F_{n+1}$ over $F_{n}$. We know that this dimension can't be greater than $d$, as this is the relative dimension of $Y_{n+1}$ over $Y_{n}$, and we also know that it is at least 1 . What we will do is show that, for any $l$, if the relative dimension of $F_{n+1}$ over $F_{n}$ is at least $l$, then we obtain a contradiction, so it must be at least $l+1$. Eventually we will get to $l=d$ where the argument must stop, and we will have to conclude that each $F_{n}$ is empty.

Assume that for all $n$ and for all $y \in F_{n}$, the dimension of the fiber above $y$ in $F_{n+1}$ is greater than or equal to $l$. Suppose there exists an element $y \in F_{n}$ with the dimension of the fiber above $y$ in $F_{n+1}$ exactly $l$. By the semicontinuity of the fiber dimension of projective maps, there is an irreducible component of $F_{n}$, call it $M_{n}$, with the properties that $M_{n}$ contains $y$, and the general fibers in $F_{n+1}$ over $M_{n}$ have dimension equal to $l$. It also follows by induction that for any integer $k$ the dimensions of the general fibers in $F_{n+k}$ above $M_{n}$ are equal to $k l$.

The next section of the paper will be devoted to proving the following proposition:

Proposition 1.15. For large values of $k$ and $n$, suppose that $F$ is a component of $F_{n+k} \backslash \Theta_{F_{n+k}}$ such that $F$ has relative dimension $k l$ over $F_{n}$ and the image of $F$ in $F_{n+1}$ has relative dimension $l$ over $F_{n}$. Then $F$ is of logarithmic general type. 
Remember, however, that by definition the set of integral points in $F_{n+k} \backslash \Theta_{F_{n+k}}$ is dense; the Lang-Vojta conjecture combined with the work above implies a contradiction. Thus we have discovered that, for all $n$ and for all $y \in F_{n}$, the dimension of the fiber above $y$ in $F_{n+1}$ must be at least $l+1$.

Continuing by induction on this relative dimension, we will be forced to conclude that the conjecture implies a contradiction, unless $F_{n+k}$ is empty for large values of $k$ and $n$, hence proving Theorem 1.1.

\section{Proof of Proposition 1.15}

By definition each $F_{n}$ is contained in $Y_{n} \backslash \Theta_{Y_{n}}$. Define $G_{n}$ to be the subvariety of $E_{n}^{d}$ which is the inverse image of $F_{n}$ under the quotient map given by the action of the symmetric group. Fix $n$. As in the proof of Lemma 4.1 in $[\mathrm{P}]$, we may replace each $G_{n+k}$, for $k \geq 0$, with an irreducible component of relative dimension $k l$ over $G_{n}$.

We have projection maps $\pi: G_{n+1} \rightarrow G_{n}$ such that $\pi\left(G_{n+1}\right)=G_{n}$. Notice also that $G_{n+k} \subseteq\left(G_{n+1}\right)_{G_{n}}^{k}$ (see the proof of Lemma 4.1 in $[\mathrm{P}]$ ).

Let $\phi$ be the projection of $G_{n}$ to $\left(\mathbf{P}^{1}\right)^{d}$, and for $i=1, \ldots, d$, let $f_{i}$ be the $d$ projections from $\left(\mathbf{P}^{1}\right)^{d}$ to $\mathbf{P}^{1}$. Let $B=\phi\left(G_{n}\right)$, and set $B_{i}=f_{i} \circ \phi\left(G_{n}\right)$, for $i=1, \ldots, d$. Notice that $B$ is irreducible since it is the image of the irreducible $G_{n}$, and $B \subset B_{1} \times \cdots \times B_{d}$, where $B$ surjects to each $B_{i}$. Finally, let $\widetilde{B}$ be a desingularization of $B$ with the property that its discriminant locus is a divisor with normal crossings. In other words, we consider the pullback of $E_{1}^{d} \rightarrow\left(\mathbf{P}^{1}\right)^{d}$ to $\widetilde{B}$, and require that the discriminant locus be a divisor of normal crossings, where the discriminat locus is the set of points in $\widetilde{B}$ which have singular fibers lying above them. Let $g_{i}: \widetilde{B} \rightarrow \mathbf{P}^{1}$ be the composition of the maps and inclusions in the diagram below:

$$
\widetilde{B} \rightarrow B \subset B_{1} \times \cdots \times B_{d} \subseteq\left(\mathbf{P}^{1}\right)^{d} \stackrel{f_{i}}{\rightarrow} \mathbf{P}^{1} .
$$

Define families of curves:

$$
C_{i}=g_{i}^{*}\left(E_{1} \rightarrow \mathbf{P}^{1}\right)=E_{1} \times \mathbf{P}^{1} \widetilde{B} .
$$

By an abuse of notation, we shall pull back all $G_{n+k}$ 's $(k \geq 0)$ to $\widetilde{B}$ and pull back the $C_{i}$ 's to $G_{n}$, but keep the notation the same. Lemma 4.1 in $[\mathrm{P}]$ shows that this new $G_{n+1}$ is contained inside the product of $d$ families of curves, $C_{1} \times_{G_{n}} \cdots \times_{G_{n}} C_{d}$, and it surjects onto each family.

From $[\mathrm{P}], \S 4.2$, we also know that there exist $d$ surjective generically finite maps, $\sigma_{i}$, from $G_{n+1}$ to a product of $l$ of the $C_{i}$ 's fibered over $G_{n}$, where $l$ is the relative dimension of $G_{n+1}$ over $G_{n}$. In particular, for each $i=1, \ldots, d$, we have

$$
\sigma_{i}: G_{n+1} \rightarrow C_{j_{1}(i)} \times_{G_{n}} \cdots \times_{G_{n}} C_{j_{l}(i)},
$$

where one of the indices $j_{k}(i)$ is equal to $i$.

There is a projection from $G_{n}$ down to $\widetilde{B}$, and we can now obtain maps from $G_{n}$ to fibered products of families of curves over $\widetilde{B}$ :

$$
G_{n} \rightarrow C_{1}^{m_{1}} \times_{\widetilde{B}} \cdots \times_{\widetilde{B}} C_{d}^{m_{d}} .
$$

Hence we can express the $\sigma_{i}$ 's as:

$$
\sigma_{i}: G_{n+1} \rightarrow C_{j_{1}(i)} \times_{\widetilde{B}} \cdots \times_{\widetilde{B}} C_{j_{l}(i)} \times_{\widetilde{B}} C_{1}^{m_{1}} \times_{\widetilde{B}} \cdots \times_{\widetilde{B}} C_{d}^{m_{d}} .
$$


We proceed as in $[\mathrm{P}], \S 4.2$; using the fact that $G_{n+k} \subseteq\left(G_{n+1}\right)_{G_{n}}^{k}$, we apply the $\sigma_{i}$ 's to the various copies of $G_{n+1}$ in the above product, obtaining a generically finite map from $G_{n+k}$ to a product of powers of the $C_{i}$ 's:

$$
G_{n+k} \rightarrow C_{1}^{k_{1}} \times_{\widetilde{B}} \cdots \times_{\widetilde{B}} C_{d}^{k_{d}} .
$$

We shall denote by $V_{n+k}$ (where $k \geq 0$ ) the product on the right above:

$$
V_{n+k}=C_{1}^{k_{1}} \times_{\widetilde{B}} \cdots \times_{\widetilde{B}} C_{d}^{k_{d}} .
$$

If we increase $k$, we can increase the above exponents $k_{i}$. For instance, in going from $G_{n+k}$ to $G_{n+k+1}$, we obtain one more copy of $G_{n+1}$, allowing us to apply one more of the $\sigma_{i}$ 's.

We now turn our attention to the divisors on these products of families of curves. This is where our work here differs from that done in $[\mathrm{P}]$. Notice that each $C_{i}$ contains the divisor $g_{i}^{*}(\Theta)$, the pullback of the origin of the surface $E_{1}$. For $i=$ $1, \ldots, d$, we may choose a projection

$$
p_{i}: C_{1} \times_{\widetilde{B}} \cdots \times_{\widetilde{B}} C_{d} \rightarrow C_{i} .
$$

Let $Z$ denote this product

$$
Z=C_{1} \times_{\widetilde{B}} \cdots \times_{\widetilde{B}} C_{d}
$$

and define a divisor $D_{Z}$ on $Z$ by

$$
D_{Z}=\sum_{i=1}^{d} p_{i}^{*} g_{i}^{*}(\Theta) .
$$

Denote by $Z_{i}$ the product

$$
Z_{i}=C_{j_{1}(i)} \times_{\widetilde{B}} \cdots \times_{\widetilde{B}} C_{j_{l}(i)},
$$

where, as above, for each $i$ one of the subscripts $j_{k}(i)$ is equal to $i$, and define the divisor $D_{Z_{i}}$ on $Z_{i}$ by

$$
D_{Z_{i}}=\sum_{k=1}^{l} p_{j_{k}(i)}{ }^{*} g_{j_{k}(i)} *(\Theta) .
$$

Let $\tau$ be the map from $G_{n+1}$ to $Z$ induced by the inclusion $G_{n+1} \subset G_{n} \times_{\widetilde{B}} Z$, and let $W$ be the image of $G_{n+1}$ in $Z$, where $e: W \hookrightarrow Z$ is the inclusion map.

Lemma 2.1. We have that

$$
e \circ \tau\left(G_{n+1} \backslash \Theta_{n+1}\right) \subset Z \backslash D_{Z} .
$$

Moreover, for each $i=1, \ldots, d$, we have dominant morphisms

$$
\sigma_{i}: G_{n+1} \backslash \Theta_{n+1} \rightarrow Z_{i} \backslash D_{Z_{i}} .
$$

Proof. The first statement follows automatically, since $(e \circ \tau)^{*}\left(D_{Z}\right) \subset \Theta_{n+1}$, and hence the complement maps to the complement as desired. For the second statement, consider the surjective maps $\sigma_{i}: G_{n+1} \rightarrow Z_{i}$. When restricted to $G_{n+1} \backslash \Theta_{n+1}$, their image is dense in $Z_{i} \backslash D_{Z_{i}}$.

For large $n$, let $V_{n}$ be previously defined product

$$
V_{n}=C_{1}^{k_{1}} \times_{\widetilde{B}} \cdots \times_{\widetilde{B}} C_{d}^{k_{d}} .
$$

Define the canonical projections

$$
r_{i}: V_{n} \rightarrow C_{i}^{k_{i}}
$$


for $i=1, \ldots, d$, and, for $j_{i}=1, \ldots, k_{i}$,

$$
q_{j_{i}}: C_{i}^{k_{i}} \rightarrow C_{i} .
$$

Then $V_{n}$ contains the divisor

$$
D_{V_{n}}=\sum_{i=1}^{d} \sum_{j_{i}=1}^{k_{i}} r_{i}^{*} q_{j_{i}}^{*} g_{i}^{*} \Theta .
$$

It follows that $G_{n} \backslash \Theta_{n}$ maps generically finitely to $V_{n} \backslash D_{V_{n}}$, and for positive $k$, $G_{n+k} \backslash \Theta_{n+k}$ maps generically finitely to $V_{n+k} \backslash D_{V_{n+k}}$.

From [A2], Lemma 3, we know that the relative dualizing sheaf $\omega_{E_{1} / \mathbf{P}^{1}}(\Theta)$ is big. This is the essential ingredient in proving the following lemma.

Lemma 2.2. For large $n$, both $\omega_{V_{n} / \widetilde{B}}\left(D_{V_{n}}\right)$ and $\omega_{V_{n}}\left(D_{V_{n}}\right)$ are big.

Proof. The proof follows exactly as in the proof of Lemma 4.5 in $[\mathrm{P}]$.

Remark 2.3. In order to apply Lemma 4.5 of $[\mathrm{P}]$, we need to know that Lemmas 3.5 and 4.4 of $[\mathrm{P}]$ still hold when line bundles of the form $L_{1} \otimes L_{2}$ are replaced by invertible sheaves of the form $L_{1}\left(D_{1}\right) \otimes L_{2}\left(D_{2}\right)$ where the $D_{i}$ 's are divisors. For these lemmas to apply to logarithmic canonical differential forms it is important that the sum of the divisors $D_{i}$ is a reduced divisor, as such forms must have simple poles along the divisor (see [I], Chapter 11). This does hold, however, as the divisors $D_{V_{n}}$ which we work with here are constructed as sums of pullbacks of horizontal divisors via distinct maps, and hence are reduced.

Let $\mathcal{G}_{k}$ be the quotient of $G_{n+k} \backslash \Theta_{n+k}$ modulo the action of the symmetric group. To prove Proposition 1.15 we need to show that $\mathcal{G}_{k}$ is of log-general type. We know from [A2], Lemma 2, that for all $n>2, V_{n} \backslash D_{V_{n}}$ has log-canonical singularities. We also know that $V_{n}$ has canonical singularities, as it is a family of nodal curves (see, for instance, Lemma 3.3 of $[\mathrm{CHM}]$ ). These two facts are important, as they show that the singularities do not prohibit the extension of sections to a desingularization. Thus we will be able to use our projections to products of families of curves to pull back log-differential forms to $G_{n+k} \backslash \Theta_{n+k}$, and to pull them back even further to a desingularization.

By Lemma 4 in [A1], what we must do is show that not only is $\omega_{G_{n+k}}\left(\Theta_{G_{n+k}}\right)$ big, but also that its sections vanish to sufficiently high order on the fixed points of the symmetric group action on $G_{n+k} \backslash \Theta_{n+k}$. This will let us conclude that any desingularization of $\mathcal{G}_{k}$ is of log-general type, and hence will prove Proposition 1.15.

By Hironaka's theorem we may choose an equivariant resolution of singularities, $\widetilde{G_{n+k}}$, of $G_{n+k}$, such that the pullback of $\Theta_{n+k}$, call it $\widetilde{\Theta_{n+k}}$, is a divisor of normal crossings. Denote by $r$ the desingularization map

$$
r: \widetilde{G_{n+k} \backslash \widetilde{\Theta_{n+k}}} \rightarrow G_{n+k} \backslash \Theta_{n+k} .
$$

Let $\pi_{0}$ denote the map from $G_{n+k} \backslash \Theta_{n+k}$ to $G_{n} \backslash \Theta_{n}$, and let $\sigma_{0}$ be the map from $G_{n} \backslash \Theta_{n}$ to $V_{n} \backslash D_{V_{n}}$. For $j=1, \ldots, k$, let $\pi_{j}$ denote the map from $G_{n+k} \backslash \Theta_{n+k}$ to $G_{n+1} \backslash \Theta_{n+1}$. Finally, recall that for each $i$ we have the map $\sigma_{i}$ from $G_{n+1} \backslash \Theta_{n+1}$ 
to $Z_{i} \backslash D_{Z_{i}}$. Thus, we have

$$
\begin{aligned}
\pi_{0}: G_{n+k} \backslash \Theta_{n+k} & \rightarrow G_{n} \backslash \Theta_{n}, \\
\sigma_{0}: G_{n} \backslash \Theta_{n} & \rightarrow V_{n} \backslash D_{V_{n}}, \\
\pi_{j}: G_{n+k} \backslash \Theta_{n+k} & \rightarrow G_{n+1} \backslash \Theta_{n+1}, \\
\sigma_{i}: G_{n+1} \backslash \Theta_{n+1} & \rightarrow Z_{i} \backslash D_{Z_{i}} .
\end{aligned}
$$

The remaining constructions and calculations are almost exactly as in $[\mathrm{P}]$, the only difference being the divisors. Define

$$
V_{1, i}=V_{n} \times_{\widetilde{B}} Z_{i},
$$

and

$$
V_{k, i}=V_{n} \times_{\widetilde{B}}\left(Z_{i}\right)_{\widetilde{B}}^{k} .
$$

Let $D_{V_{1, i}}$ and $D_{V_{k, i}}$ be the usual divisors on $V_{1, i}$ and $V_{k, i}$, respectively.

Recall the fact that

$$
G_{n+k} \backslash \Theta_{n+k} \subseteq\left(G_{n+1} \backslash \Theta_{n+1}\right)_{G_{n} \backslash \Theta_{n}}^{k} .
$$

We use this to define a map $\left(\sigma_{0}, \sigma_{i}, \ldots, \sigma_{i}\right)$ from $G_{n+k} \backslash \Theta_{n+k}$ to $V_{k, i} \backslash D_{V_{k, i}}$ in the following manner: apply $\sigma_{0}$ to $G_{n}$, and apply the $\sigma_{i}$ 's to the copies of $G_{n+1}$. In other words, if

$$
P=\left(P_{1}, \ldots, P_{n}, P_{n+1}, \ldots, P_{n+k}\right) \in G_{n+k} \backslash \Theta_{n+k},
$$

then

$$
\left(\sigma_{0}, \sigma_{i}, \ldots, \sigma_{i}\right)(P)=\left(\sigma_{0}\left(P_{1}, \ldots, P_{n}\right), \sigma_{i}\left(P_{n+1}\right), \ldots, \sigma_{i}\left(P_{n+k}\right)\right) .
$$

Define projections $\rho_{i}: Z \rightarrow Z_{i}$

$$
\rho_{i}\left(C_{1} \times_{\widetilde{B}} \cdots \times_{\widetilde{B}} C_{d}\right)=C_{j_{1}(i)} \times_{\widetilde{B}} \cdots \times_{\widetilde{B}} C_{j_{l}(i)},
$$

where we also have

$$
\rho_{i}: Z \backslash D_{Z} \rightarrow Z_{i} \backslash D_{Z_{i}} .
$$

Let $\Phi_{n+1}$ and $\Phi_{n+k}$ be the locus of points fixed by the group action in $G_{n+1} \backslash \Theta_{n+1}$ and $G_{n+k} \backslash \Theta_{n+k}$, respectively. It is important to note that the image in $W$ of the fixed points $\Phi_{n+1}$ is not all of $W$. This follows by the same argument as in Lemma 4.2 of $[\mathrm{P}]$.

From Lemma 2.2 we know that $\omega_{V_{n}}\left(D_{V_{n}}\right)$ is big; it also follows that $\omega_{V_{k, i}}\left(D_{V_{k, i}}\right)$ is big, since the families of curves appear in $V_{k, i}$ with even higher exponents than in $V_{n}$.

To finish the proof of Proposition 1.15, we use the fact that $\omega_{V_{k, i}}\left(D_{V_{k, i}}\right)$ is big to pull back sections to $G_{n+k} \backslash \Theta_{n+k}$ and to $\widetilde{G_{n+k} \backslash \widetilde{\Theta_{n+k}}}$, and then show that these sections vanish to sufficiently high order on the locus of fixed points. Unfortunately, we can't simply pull back sections along any one projection; to guarantee that the sections we pull back will vanish along the fixed points, we must pull back sections via many different projections and tensor them together.

If we pull back sections of $\omega_{V_{k, i}}\left(D_{V_{k, i}}\right)$, we see that

$$
r^{*}\left(\left(\sigma_{0}, \sigma_{i}, \ldots, \sigma_{i}\right)^{*} \omega_{V_{k, i}}\left(D_{V_{k, i}}\right)\right) \hookrightarrow \omega \widetilde{G_{n+k}}\left(\widetilde{\Theta_{n+k}}\right) .
$$


Moreover, if we tensor over all $i=1, \ldots, d$, we have that

$$
r^{*}\left(\bigotimes_{i}\left(\sigma_{0}, \sigma_{i}, \ldots, \sigma_{i}\right)^{*} \omega_{V_{k, i}}\left(D_{V_{k, i}}\right)\right) \hookrightarrow \omega_{\widetilde{G_{n+k}}}^{\otimes d}\left(\widetilde{d \Theta_{n+k}}\right) .
$$

Let's rewrite the left-hand side above. We can break it down into the portion which comes from $V_{n}$ and the portion which comes from the powers of $Z_{i}$ :

$$
\begin{gathered}
r^{*}\left(\bigotimes_{i}\left(\sigma_{0}, \sigma_{i}, \ldots, \sigma_{i}\right)^{*} \omega_{V_{k, i}}\left(D_{V_{k, i}}\right)\right) \\
=r^{*} \pi_{0}^{*} \sigma_{0}^{*} \omega_{V_{n}}^{\otimes d}\left(d D_{V_{n}}\right) \otimes r^{*} \pi_{1}^{*}\left(\bigotimes_{i} \sigma_{i}^{*} \omega_{Z_{i} / \widetilde{B}}\left(D_{Z_{i}}\right)\right) \otimes \cdots \otimes r^{*} \pi_{k}^{*}\left(\bigotimes_{i} \sigma_{i}^{*} \omega_{Z_{i} / \widetilde{B}}\left(D_{Z_{i}}\right)\right) .
\end{gathered}
$$

Let's isolate and expand one of the last $k$ terms in the above product. We shall use the fact that $\sigma_{i}=\rho_{i} \circ e \circ \tau$; hence $\sigma_{i}^{*}=\tau^{*} e^{*} \rho_{i}^{*}$. Letting $1 \leq j \leq k$, we see that:

$$
\begin{aligned}
& r^{*} \pi_{j}^{*}\left(\bigotimes_{i} \sigma_{i}^{*} \omega_{Z_{i} / \widetilde{B}}\left(D_{Z_{i}}\right)\right)=r^{*} \pi_{j}^{*}\left(\bigotimes_{i} \tau^{*} e^{*} \rho_{i}^{*} \omega_{Z_{i} / \widetilde{B}}\left(D_{Z_{i}}\right)\right) \\
& =r^{*} \pi_{j}^{*}\left(\bigotimes_{i}\left(\tau^{*} e^{*}\left(\omega_{C_{j_{1}(i) / \widetilde{B}}}\left(D_{C_{j_{1}(i)}}\right) \otimes \cdots \otimes \omega_{C_{j_{l}(i) / \widetilde{B}}}\left(D_{C_{j_{l}(i)}}\right)\right)\right)\right) \\
& =r^{*} \pi_{j}^{*} \tau^{*} e^{*}\left(\bigotimes_{i}\left(\omega_{C_{j_{1}(i) / \widetilde{B}}}\left(D_{C_{j_{1}(i)}}\right) \otimes \cdots \otimes \omega_{{j_{l}(i) / \widetilde{B}}}\left(D_{C_{j_{l}(i)}}\right)\right)\right) \\
& =r^{*} \pi_{j}^{*} \tau^{*} e^{*}\left(\omega_{C_{1} / \widetilde{B}}^{\otimes_{l_{1}}}\left(l_{1} D_{C_{1}}\right) \otimes \cdots \otimes \omega_{C_{d} / \widetilde{B}}^{\otimes_{l_{d}}}\left(l_{d} D_{C_{d}}\right)\right)
\end{aligned}
$$

where

$$
D_{C_{j_{1}(i)}}=p_{j_{1}(i)}^{*} g_{j_{1}(i)}^{*} \Theta \quad, \ldots, \quad D_{C_{j_{l}(i)}}=p_{j_{l}(i)}^{*} g_{j_{l}(i)}^{*} \Theta
$$

and for $i=1, \ldots, d$,

$$
D_{C_{i}}=p_{i}^{*} g_{i}^{*} \Theta .
$$

The exponents $l_{1}, \ldots, l_{d}$ are defined by the last line above, and each one is positive. The idea is that for each $i$ we know that one of the $j_{k}(i)$ 's is equal to $i$. Thus in the last line, when we tensor over all $i$, each $\omega_{C_{1} / \widetilde{B}}\left(l_{1} D_{C_{1}}\right)$ appears at least once, and we let $l_{i}$ indicate the number of times it appears.

Let $M$ denote the line bundle $\omega_{C_{1} / \widetilde{B}}^{\otimes l_{1}}\left(l_{1} D_{C_{1}}\right) \otimes \cdots \otimes \omega_{C_{d} / \widetilde{B}}^{\otimes l_{d}}\left(l_{d} D_{C_{d}}\right)$, and let $M_{W}$ stand for $e^{*} M$, the pullback of $M$ to $W$. By similar reasoning as in Lemma 3.5 of [P], we know that $M$ is big on $Z$. Since $W$ is the image of $G_{n+1} \backslash \Theta_{n+1}$ in $Z \backslash D_{Z}$, it follows that $W$ surjects to each $C_{i} \backslash D_{C_{i}}$. By Lemma 3.5 in [P] this implies that $M_{W}$ is big on $W$.

We now know that

$$
r^{*} \pi_{0}^{*} \sigma_{0}^{*} \omega_{V_{n}}^{\otimes d}\left(d D_{V_{n}}\right) \otimes r^{*} \pi_{1}^{*} \tau^{*} M_{W} \otimes \cdots \otimes r^{*} \pi_{k}^{*} \tau^{*} M_{W} \hookrightarrow \omega \widetilde{G_{n+k}}\left(\widetilde{d \Theta_{n+k}}\right) .
$$

Moreover, each term above is big. Recall that the image of the fixed points $\Phi_{n+1}$ in $W$ is not equal to all of $W$; hence the image forms a proper subvariety. This means that we obtain a non-zero ideal of sections of $M_{W}$ which vanish on this subvariety. Call this ideal $\mathcal{I}$. 
We borrow the following previously mentioned result from [A2], a result which generalizes Lemma 4.1 of $[\mathrm{CHM}]$ :

Lemma 2.4 (See [A2], Lemma 4). Let $X_{0} \subset X$ be an open inclusion of an irreducible variety $X_{0}$ in a smooth complex projective irreducible $X$ of dimension $n$, such that the complement $D=X \backslash X_{0}$ is a divisor of normal crossings. Let $G$ be a finite group acting on $X, X_{0}, D$ compatibly. Let $\omega$ be a $G$-equivariant logarithmic $k$-canonical form on $X_{0}$. If at any point $x$ of $X_{0}$ which is fixed by some element in $G$, the form $\omega$ vanishes to order at least $C=k(|G|-1)$, then $\omega$ descends to a regular logarithmic $k$-canonical form on any desingularization of $X_{0} / G$.

We use this to show that log-differential forms on $\widetilde{G_{n+k} \backslash \widetilde{\Theta_{n+k}}}$ will descend to smooth forms modulo the group action if they vanish to a prescribed order on the fixed point locus in $\widetilde{G_{n+k}} \backslash \widetilde{\Theta_{n+k}}$.

By Lemma 4.4 of $[\mathrm{P}]$, we know that, for some sufficiently large integer $s$, the line bundle $M_{W}^{\otimes s} \otimes \mathcal{I}$ is big on $W$. Let $\mathcal{I}_{n+k}$ be the ideal consisting of the locus of fixed points in $G_{n+k} \backslash \Theta_{n+k}$. Then

$$
\sum_{j=1}^{k} \pi_{j}^{-1} \tau^{-1} \mathcal{I} \subset \mathcal{I}_{n+k}
$$

and

$$
\prod_{j=1}^{k} \pi_{j}^{-1} \tau^{-1} \mathcal{I} \subset \mathcal{I}_{n+k}^{k}
$$

Hence,

$$
\begin{gathered}
r^{*} \pi_{0}^{*} \sigma_{0}^{*} \omega_{V_{n}}^{\otimes d s}\left(d s D_{V_{n}}\right) \otimes r^{*}\left(\bigotimes_{j=1}^{k} \pi_{j}^{*} \tau^{*}\left(M_{W}^{\otimes s} \otimes \prod_{j=1}^{k} \pi_{j}^{-1} \tau^{-1} \mathcal{I}\right)\right) \\
\hookrightarrow \omega \widetilde{G_{n+k}}\left(d s \widetilde{\Theta_{n+k}}\right) \otimes \mathcal{I}_{n+k}^{k} .
\end{gathered}
$$

We know that $\omega_{V_{n}}^{\otimes d s}\left(d s D_{V_{n}}\right)$ is big, and as in the proof of Lemma 4.4 in [P], for $k>s$, we have that $M_{W}^{\otimes s} \otimes \prod_{j=1}^{k} \pi_{j}^{-1} \tau^{-1} \mathcal{I}$ is big. Thus for $k>s$ the entire left-hand side is big, so there are a sufficiently high number of sections of $\omega_{\widetilde{G_{n+k}}}^{\otimes d s}\left(\widetilde{\Theta_{\Theta_{n+k}}}\right) \otimes$ $\mathcal{I}_{n+k}^{k}$ vanishing to sufficiently high order on the fixed points.

This lets us conclude that any desingularization of the quotient $\widetilde{G_{n+k} \backslash} \widetilde{\Theta_{n+k}}$ modulo the symmetric group action is of log-general type, which implies Proposition 1.15 .

\section{REFERENCES}

[A1] D. Abramovich. Uniformité des points rationnels des courbes algébriques sur les extensions quadratiques et cubiques, C.R. Acad. Sci. Paris, t. 321, Série I (1995), p. 755-758. MR 96g:14017

[A2] D. Abramovich. Uniformity of stably integral points on elliptic curves, Invent. Math. 127 (1997), p. 307-317. MR 98d:14033

[CHM] L. Caporaso, J. Harris, and B. Mazur. Uniformity of rational points, J. Amer. Math. Soc. 10 no. 1 (1997), p. 1-35. MR 97d:14033

[I] S. Iitaka. Algebraic Geometry: an Introduction to Birational Geometry of Algebraic Varieties, Springer-Verlag, (1982). MR 84j:14001 
[KM] S. Kamienny and B. Mazur. Rational torsion of prime order in elliptic curves over number fields, Astrisque no. 228 (1995), p. 81-100. MR 96c:11058

[L] S. Lang. Hyperbolic diophantine analysis, Bull. A.M.S. 14 (1986), p. 159-205. MR 87h:32051

[M] L. Merel. Bornes pour la torsion des courbes elliptiques sur les corps de nombres, Invent. Math. 124 (1996), p. 437-449. MR 96i:11057

[P] P. Pacelli. Uniform boundedness for rational points, Duke Math. J. vol. 88 no. 1 (1997), p. 77-102. MR 98b: 14020

[RS] K. Rubin and A. Silverberg. Families of elliptic curves with constant mod p representations, Elliptic curves, modular forms, and Fermat's last theorem (Hong Kong, 1993), Ser. Number Theory, I, Internat. Press, Cambridge, MA. (1995), p. 148-161. MR 96j:11078

[S] J. H. Silverman. The Arithmetic of Elliptic Curves, Springer-Verlag, 1986. MR 87g:11070

[V] P. Vojta. A higher dimensional Mordell conjecture, in Arithmetic Geometry, (G. Cornell and J. H. Silverman, eds.), Springer-Verlag, (1986). CMP 19:03

Department of Mathematics, Barnard College, Columbia University, New York, NEW York 10027-6598

E-mail address: pacelli@math.columbia.edu 